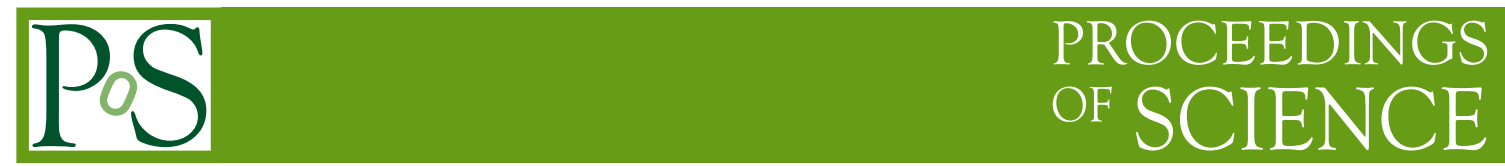

\title{
Issues in Galaxy Formation 2014
}

\section{Joe Silk*}

Institut d'Astrophysique de Paris

E-mail: silkeiap.fr

\begin{abstract}
The origin of the galaxies represents an important focus of current cosmological research, both observational and theoretical. Its resolution involves a comprehensive understanding of star formation and evolution, galaxy dynamics, supermassive black holes, and the cosmology of the very early universe. I will review our current understanding of galaxy formation and describe some of the challenges that lie ahead. Specific issues that I will address include the star formation rate in galaxies and the galaxy luminosity function, including the role of feedback.
\end{abstract}

Frontiers of Fundamental Physics 14 - FFP14,

15-18 July 2014

Aix Marseille University (AMU) Saint-Charles Campus, Marseille

\footnotetext{
${ }^{*}$ Speaker.
} 